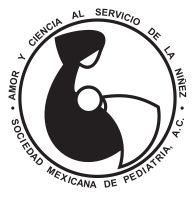

\title{
Catarata congénita neonatal unilateral
}

\author{
Luis Ángel Bolio-Molina, ${ }^{\ddagger} *$ Gabriela Toledo-Verónico ${ }^{\S}$ \\ ₹ Pediatra de práctica privada y en el Hospital General de Cuernavaca, Morelos; \\ $\S$ Diplomada en Pediatría en práctica privada. Cuernavaca, Morelos, México.
}

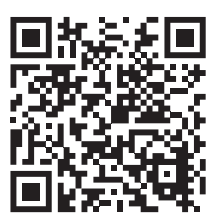

\begin{abstract}
RESUMEN
Objetivo: Dar a conocer el caso de una recién nacida (RN) con catarata congénita, lo cual es infrecuente (de uno a 15 casos por 10,000 RN), y en muchos casos el diagnóstico se retrasa. Presentación del caso: RN femenino de 16 días de edad, de término, producto de un embarazo normal. No hay antecedentes personales ni familiares relevantes. Su madre notó "mayor brillo" en su ojo izquierdo desde el tercer día de vida. A la exploración física, la única anormalidad detectada fue hiporreflexia pupilar izquierda, además de signo de reflejo rojo asimétrico y opacidad "centropupilar". Oftalmología pediátrica confirmó diagnóstico de catarata congénita, la cual se extirpó a los tres meses de edad. Aunque su evolución posterior fue satisfactoria, desarrolló glaucoma. Conclusión: La catarata congénita es un problema ocular de difícil diagnóstico, por lo que se requiere conocimiento y capacitación para su detección; la búsqueda sistemática del reflejo rojo en todo RN y lactante puede ayudar a su identificación.
\end{abstract}

Palabras clave: Catarata congénita, reflejo rojo, recién nacido, lactante.

\section{INTRODUCCIÓN}

La catarata congénita (CC) es la opacidad del cristalino presente desde el nacimiento. En países desarrollados ocurre en 1-3/10,000 recién nacidos $(\mathrm{RN})$, mientras que en países en vías de desarrollo en 1-15/10,000 RN, y constituye de 10 a $39 \%$ de la etiología de ceguera

\footnotetext{
* Correspondencia: LABM, bmolinala@hotmail.com

Conflicto de intereses: Los autores declaran que no tienen. Citar como: Bolio-Molina LA, Toledo-Verónico G. Catarata congénita neonatal unilateral. Rev Mex Pediatr. 2020; 87(1):18-20. doi: $10.35366 / 93263$

[Congenital unilateral neonatal cataract]
}

\begin{abstract}
Objective: To report the case of a newborn with congenital cataract, which is rare condition (from one to 15 cases per 10,000 newborns) and in many cases, there is a delay in diagnosis. Case presentation: 16-day-old female full-term newborn, with normal pregnancy. There was no relevant personal or family history. From the third day of life, her mother noticed "greater brightness" in her left eye. The only abnormality detected on physical examination was left pupillary hyporeflexia, in addition to an asymmetric red reflex sign and "centropupillary" opacity. A pediatric ophthalmologist confirmed the diagnosis of congenital cataract and removed it at three months of age. Although her subsequent evolution was satisfactory, she developed glaucoma. Conclusion: Congenital cataract is an eye problem that is difficult to diagnose, therefore knowledge and training are required for its detection. For its identification, the deliberate search for the red reflex in all newborns and infants is required.
\end{abstract}

Keywords: Congenital cataract, red reflex, newborn, infant.

infantil. Las causas más frecuentes de CC son rubéola congénita y avitaminosis $\mathrm{A}$.

La CC puede ser unilateral o bilateral. La más frecuente es la bilateral sin que se identifique alguna causa, aunque se puede asociar a historia familiar, síndromes, desórdenes sistémicos o infecciones. Por su parte, las unilaterales suelen ser esporádicas, sin historia familiar o alteración sistémica asociada a defectos oculares, pudiendo afectar a RN sanos. La etiología hereditaria representa 8-28\% de la CC bilateral frente a $2 \%$ de la unilateral. ${ }^{1-3}$

Se presenta el caso de una RN con CC unilateral, dado que es un padecimiento infrecuente y de difícil diagnóstico, aunado a que en los últimos años existen pocos reportes de casos similares. 


\section{PRESENTACIÓN DEL CASO}

$\mathrm{RN}$ de sexo femenino, de 16 días de vida extrauterina, producto de segundo embarazo sin complicaciones y con adecuado control prenatal. Madre de 23 años de edad, casada, comerciante, de nivel socioeconómico bajo y bachillerato incompleto. Hermana de siete años de edad, aparentemente sana.

La paciente nació a las 39 semanas de gestación por parto eutócico con peso $2.8 \mathrm{~kg}$, talla $48 \mathrm{~cm}$, perímetro cefálico $34 \mathrm{~cm}$, segmento inferior $16 \mathrm{~cm}$, calificada con Apgar 8/9. Fue egresada en las primeras 12 horas de vida, con indicación de alimentación exclusiva al seno materno.

La madre notó que el ojo izquierdo de su hija "brillaba más" (sic) que el derecho, por lo cual acudió a su centro de salud a los tres días de vida. Regresó 10 días después por persistir el síntoma, sin detectar alguna anormalidad. Dado lo anterior, deciden llevarla a un pediatra particular a los 16 días de vida.

La exploración física no reveló ninguna alteración, con excepción de que el ojo izquierdo parecía ligeramente más pequeño que el contralateral, con un "brillo especial", tendiendo a la opacidad. Con lámpara convencional se observó reflejo fotomotor izquierdo disminuido, lo que motivó explorarla con oftalmoscopio directo, lográndose detectar una opacidad "centropupi-

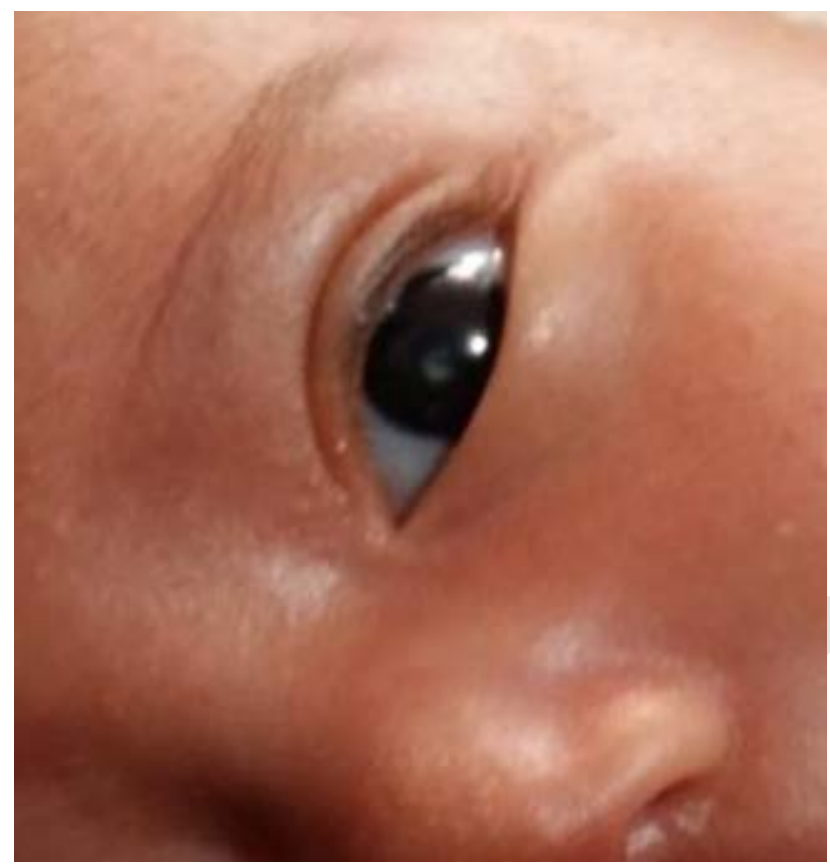

Figura 1: Catarata en ojo izquierdo.

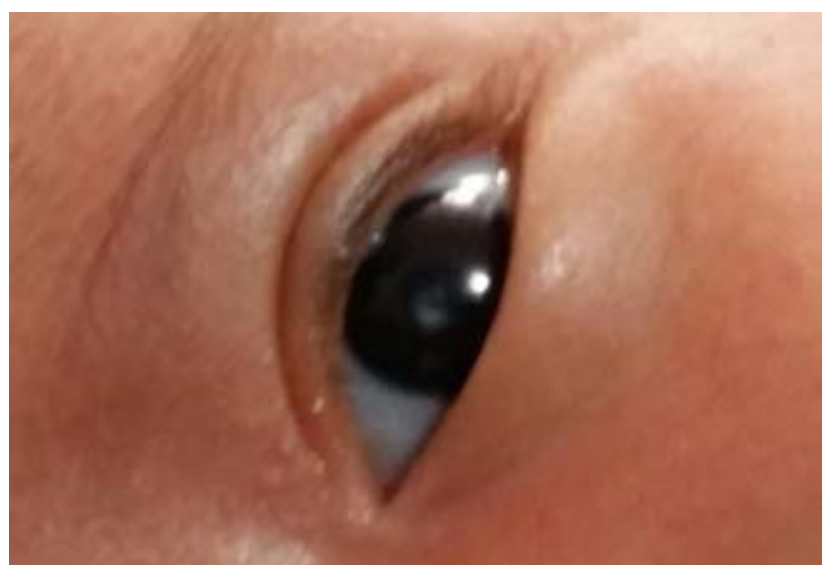

Figura 2: Acercamiento de catarata congénita neonatal.

lar" blanca, opaca y semicircular que impedía visualizar la retina, por lo que se sospechó CC (Figuras 1 y 2). Fue derivada a oftalmología del Hospital Pediátrico de Cuernavaca, Morelos, México, donde confirmaron el diagnóstico.

Cabe señalar que la serología para sífilis, toxoplasmosis, rubéola, citomegalovirus, y hepatitis infecciosa fue negativa. También se descartó que la causa fuera traumática o asociada con algún síndrome. El diagnóstico fue de CC unilateral aislada e idiopática, no meritoria de estudios de imagen ni valoración genética.

Fue operada a los tres meses de edad, pero el tamaño ocular impidió implantarle lente intraocular. Desarrolló glaucoma de ángulo cerrado ameritando válvula de derivación a los seis meses de edad. Actualmente tiene 24 meses, con desarrollo físico y neurológico acorde con su edad cronológica, y agudeza visual disminuida del ojo afectado.

\section{DISCUSIÓN}

Para detectar defectos oculares, la Academia Americana de Pediatría recomienda explorar el "reflejo rojo" (RR) o prueba de Bruckner desde el nacimiento, así como durante las consultas de seguimiento pediátrico en el transcurso del primer año de vida. Sin embargo, por falta de capacitación de los médicos de primer contacto en la realización de esta prueba no se hace de manera rutinaria..$^{1-4}$ Por lo anterior, se retrasa su detección, diagnóstico y tratamiento.

$\mathrm{Al}$ respecto, en la República Popular China dado que un número significativo de niños no eran diagnosticados, se dio capacitación sobre el examen ocular neonatal para identificar enfermedades oculares graves en todos 
sus RN sanos. Con esta estrategia se lograron tasas de exploración ocular de $75.5 \%(1,881$ de $2,490 \mathrm{RN})$ a los siete meses de edad y de $93.9 \%$ a los 11 meses. $^{5}$

El motivo de consulta depende del grado de densidad de la catarata, del momento de aparición y de si es unilateral o bilateral. ${ }^{6}$ Creemos que también depende de la edad del paciente, de que la madre sea observadora, pero sobre todo de que el médico escuche, crea y tome en cuenta lo que las madres nos comentan.

La presentación clínica varía con la edad. Cuando hay leucocoria (o pupila blanca) y nistagmo en cataratas densas puede facilitar el diagnóstico. En lactantes de tres meses de edad, en catarata bilateral existe dificultad del contacto visual, mala fijación, falta de seguimiento visual, así como ausencia del RR. En lactantes de un año o mayores se puede presentar estrabismo, disminución de la agudeza visual y retardo del aprendizaje. La madre es la primera en notar anormalidades como agudeza visual disminuida, inclinación y desviación de la cabeza, rendimiento escolar deficiente, o malas posturas. ${ }^{6}$ En nuestra paciente la madre notó "mayor brillo" del ojo afectado, lo cual no se describe en la literatura.

La CC causa compromiso visual por ambliopía profunda e irreversible que depende de factores como: lateralidad, densidad, tamaño y localización de la opacidad. Si interfiere con el campo visual está indicado su tratamiento, el cual es controversial; sin embargo, si es oportuno y con rehabilitación mejoran los resultados visuales. ${ }^{7}$

En México se recomienda la realización del tamiz oftalmológico neonatal a la cuarta semana del nacimiento para la detección temprana y en su caso, tratamiento de malformaciones que puedan causar ceguera. Sin embargo, la norma técnica oficial no describe cómo realizar dicho tamiz. ${ }^{8}$

La detección de CC por ultrasonografía prenatal es posible y ayuda a desarrollar el plan de manejo del neonato. ${ }^{1,9}$ Sin embargo, el diagnóstico postnatal sigue siendo un desafío, incluso en países desarrollados. El retraso en el tratamiento quirúrgico conduce a alteraciones de la función visual y, en consecuencia, en el área motora, cognitiva y del lenguaje, limitando el desarrollo integral y adecuado en el individuo. ${ }^{10}$

\section{REFERENCIAS}

1. De Diego AE, Savirón CR, Odriozola GM, Lerma PD, Corbacho GT, Pérez ÍM. Diagnóstico prenatal de catarata congénita. Casos clínicos. Rev Chil Obstet Ginecol. 2013; 78(6): 455-459.

2. Khokhar S, Jose CP, Sihota R, Midha N. Unilateral congenital cataract: clinical profile and presentation. J Pediatr Ophthalmol Strabismus. 2018; 55(2): 107-112.

3. Committee on Practice and Ambulatory Medicine, Section on Ophthalmology, American Association of Certified Orthoptists, American Association for Pediatric Ophthalmology and strabismus, American academy of ophthalmology. Visual system assessment in infants, children, and young adults by pediatricians. Pediatrics. 2016; 137(1): 28-30.

4. Donahue SP, Nixon CN, Committee on Practice and Ambulatory Medicine, Section on Ophthalmology, American Association of Certified Orthoptists, American Association for Pediatric Ophthalmology and Strabismus, American Academy of Ophthalmology. Procedures for the Evaluation of the Visual System by Pediatricians. Pediatrics. 2016; 137(1): 1-9.

5. Li LH, Li N, Zhao JY, Fei P, Zhang G, Mao J, Rychwalski P. Findings of perinatal ocular examination performed on 3573 , healthy full-term newborns. Br J Ophthalmol. 2013; 97: 588-591.

6. López-Torres V, Salamanca-Libreros OF, Törnquist AL. Recomendaciones para el examen visual en los niños. latreia. 2019; 32(1): 40-51.

7. Pérez-Pérez JF, Arrollo Yllanes ME, Murillo-Murillo L. Manejo de la catarata congénita: experiencia en el Hospital General de México. Rev Mex Oftalmol. 2005; 79(3): 139-144.

8. Juárez-Echenique JC. Tamizado oftalmológico neonatal. Criterio pediátrico. Acta Pediatr Mex. 2015; 36: 361-363.

9. Lee JO, Lee JJ, Kim MJ, Lee HJ, Lee DH, Kim JS et al. Prenatal ultrasonographic diagnosis of congenital cataract: a case report. Korean J Obstet Gynecol. 2012; 55(11): 839-842.

10. Uribe-Campos L, Arroyo-Muñoz L, Mandujano-Valdez M, MorenoMacías H, Centeno-Ruíz JC, Muñoz-Hernández R. Catarata congénita bilateral: ambliopía por deprivación y su relación con el desarrollo. Rev Mex Oftalmol. 2018; 92(4): 191-200. 